\title{
EVALUATION OF THE EFFICACY OF LEUKOCYTE-PLATELET RICH FIBRIN VERSUS PLATELET RICH FIBRIN MATRIX ON BONE HEALING
}

\author{
Mohammad Hassan Nour* and Reham Lotfy Aggour **
}

\begin{abstract}
Objective: The aim of this study was to compare the efficacy of leukocyte-platelet-rich fibrin (L-PRF) and platelet-rich fibrin matrix (PRFM) on bone healing.

Methods: Eight rabbits were included in this controlled, prospective, blinded study. Defects with a diameter of $3 \mathrm{~mm}$ and $2 \mathrm{~mm}$ depth were created in the bone of both tibias, filled with L-PRF (group II), PRFM (group III) or left empty (group I). The bone mineral density (BMD) was measured by dual energy absorptiometry (DXA) and histological examination was performed on the 10 th and 21 st days.
\end{abstract}

Results: According to Emery's histopathological healing criteria, control group (group I) revealed score 0 and score 3 at 10 and 21 days respectively. Group II showed score 2 and score 5 at 10 and 21 days respectively. On the other side group III treated by PRFM showed score 3 and score 7 at 10 and 21 days respectively.

Conclusion: Both L-PRF and PRFM showed increased bone formation in surgically created bone defects in rabbits, however, the effect of PRFM was more pronounced. The use of PRFM and L-PRF may contribute to the success of bone regeneration.

KEYWORDS: Bone regeneration, L-platelet-rich fibrin, platelet-rich fibrin matrix.

\section{INTRODUCTION}

Bone tissue regeneration is a complex process involving a number of cellular functions and mineralization of the defect followed by an eventual remodeling of the defect site to reconstruct the original structure ${ }^{(1)}$. Several studies have shown that bone regenerative procedures may be enhanced by the addition of specific growth factors ${ }^{(2,3)}$ and the use of biomaterials for guided tissue/bone regeneration ${ }^{(4)}$.

* Lecturer of Oral and maxillofacial surgery, Faculty of Dentistry, October 6 University

** Associate professor of Oral Medicine, Periodontology, Oral Diagnosis and Oral Radiology, Faculty of Dentistry, October 6 University 
Platelet-rich fibrin (PRF) was introduced by Dohan et al. as a second-generation platelet concentrate ${ }^{(5)}$. In a simple preparation technique, blood is collected without anticoagulant or thrombin and immediately centrifuged only once. The growth factors present in PRF are the same as those in platelet rich plasma (PRP). Kawase et al. ${ }^{(6)}$ suggested that a fibrous network of insoluble fibrin provides a scaffold for cells and serves as a substrate for the sustained release of growth factors. A previous study demonstrated that fibrin provides a matrix for cell growth and differentiation and provide a preferable environment for osteoblastic differentiation, during which cells contact fibrin molecules and exhibit three-dimensional cell-cell interactions ${ }^{(7)}$. Moreover, PRF has played a role in space making for bone regeneration. PRF in a compressed membrane-like form has also been used as a substitute for commercially available barrier membranes in guided-tissue regeneration (GTR) treatment. However, the PRF membrane is resorbed within 2 weeks or less at implantation sites; therefore, it can barely maintain sufficient space for bone regeneration ${ }^{(8)}$.

Lucarelli et al ${ }^{(9)}$ showed that the mechanical properties of PRF can be improved by inducing extensive fibrin network formation through increased gravitational force through high-speed centrifugation of intact platelets and fibrin in the absence of exogenous thrombin. With this procedure, platelet rich plasma (PRP) can be produced in the form of platelet-rich fibrin matrix (PRFM). The PRFM preparation process creates an ideal scaffold that contains high concentrations of nonactivated, functional, intact platelets, contained within a dense cross-linked fibrin matrix. Carroll et al. ${ }^{[10]}$ have demonstrated, in vitro, that the viable platelets in PRFM released 6 growth factors in about the same concentration for the 7-day duration of their study. The PRFM membrane could be of great potential benefit in both soft tissue and bone repair.
Therefore, the aim of this experimental study was to evaluate and compare the osteogenic effects of L-PRF and PRFM in surgically created bone defects of rabbit tibia.

\section{METHODS}

The study was conducted between January 2017 and March 2017 in accordance with the Declaration of Helsinki of 1964, revised in Tokyo 2004 and was approved by the institutional ethics committee. The study included eight rabbits (weight, $2.5-3 \mathrm{~kg}$ ). Two preoperative intravenous samples of $4.5 \mathrm{~cm}^{3}$ of blood were taken from the veins of the ear of each rabbit and the blood samples were centrifuged to produce autologous L-PRF and PRFM.

\section{L-PRF Preparation}

PRF was prepared according to the technique described by Dohan et al. ${ }^{(5)}$. Immediately after 4.5 cc of blood had been drawn from the veins of the ear of each rabbit, the blood sample is centrifuged in a 5-mL sterile tube (without anticoagulant) at $400 \mathrm{~g}$ for $10 \mathrm{~min}$. L-PRF layer is located between the red corpuscles at the bottom of the tube and acellular plasma at the top of the tube [Fig. 1 (a)]. The L-PRF was thus removed from the tube using sterile tweezers and pressed between two pieces of surgical gauze to obtain the membrane form.

\section{PRFM Preparation}

PRFM was prepared according to the manufacturer's instructions (FIBRINET®, Cascade Medical Enterprises, and Wayne, NJ, USA). Briefly, $4.5 \mathrm{~cm}^{3}$ of blood was collected into sterile supplied citrate containing tubes. After gentle mixing, the tubes were centrifuged at $1100 \mathrm{~g}$ for 6 minutes to obtain PRP. Under sterile conditions, PRP was transferred into a calcium chloride-containing tube $(0.25 \mathrm{~mL} \mathrm{CaCl} 21 \mathrm{M})$. The tube was gently swirled and then immediately centrifuged at $4500 \mathrm{~g}$ for 25 minutes at room temperature. A translucent, yellowwhite PRFM was recovered at the bottom of the tube (9) [Fig. 1 (b)]. 


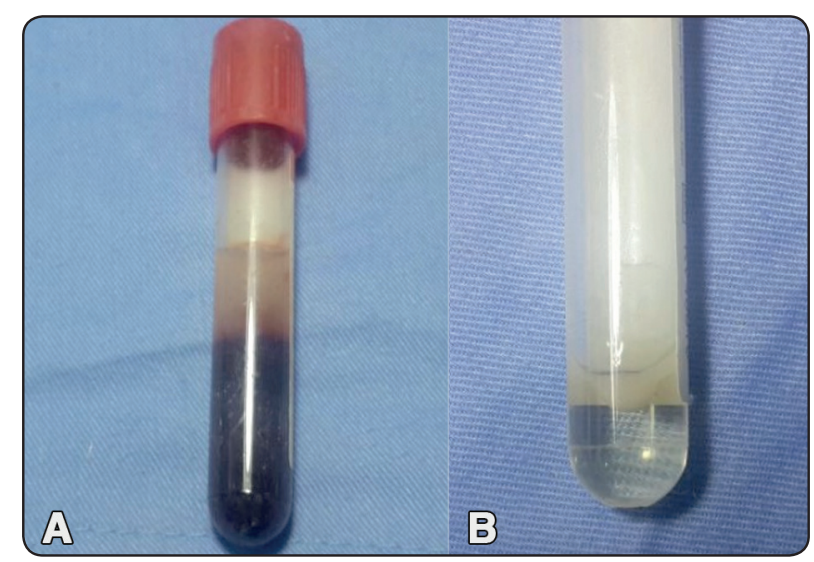

Fig (1) a) Obtaining L-PRF. b) Obtaining PRFM

\section{Experimental groups}

Eight rabbits were included in this blinded prospective study. The animals were divided into two experimental groups (Group A and Group B) ( $\mathrm{n}=4$ animals/ group). Group A animals was sacrificed after 10 days and Group B animals was sacrificed 21 days after surgery. The animals were operated under intravenous general anaesthesia. Both tibias were shaved and cleansed with $10 \%$ povidone iodine before manipulation. After a skin incision in the tibial area, the subcutaneous tissue was dissected down to the periosteum and a periosteal incision was made. In both groups, two defects with a diameter of $3 \mathrm{~mm}$ and $2 \mathrm{~mm}$ depth were osteotomized in the right tibial bone, and one defect with the same dimensions was osteotomized in the left tibial bone in the same rabbit. Sterile saline at $1500 \mathrm{rpm}$ was irrigated over the drilling site during the procedure. The upper defect of the right tibia received L-PRF. The other defect on the same tibia left empty (control group) [Fig. 2 (a)]. The defect of the left tibia received PRFM [Fig. 2 (b)]. After obtaining adequate hemostasis, the periosteum and the muscle were sutured using 4/0 polyglycolic acid sutures, and the skin with $3 / 0$ silk sutures. Antibiotics (Gentamycin $5 \mathrm{mg} / \mathrm{kg}$, Sigma Chemicals, USA) were injected intramuscularly to all rabbits to prevent infection. All efforts were made to minimize pain or discomfort of the animals.

\section{Bone Mineral Density Analysis}

In order to measure bone mineral density (BMD), we used specimens harvested at 10 and 21 days (4 animals at each time interval). A total body dual-energy X-ray (DXA) scanner (DPX, Lunar, Madison, WI, USA) and software which was tailored for small animals (Norland, Small Animal Software, 1.0c, Lunar) were used. The operated tibia were examined by the DXA scanner. Regional BMD measurements were obtained by placing rectangular

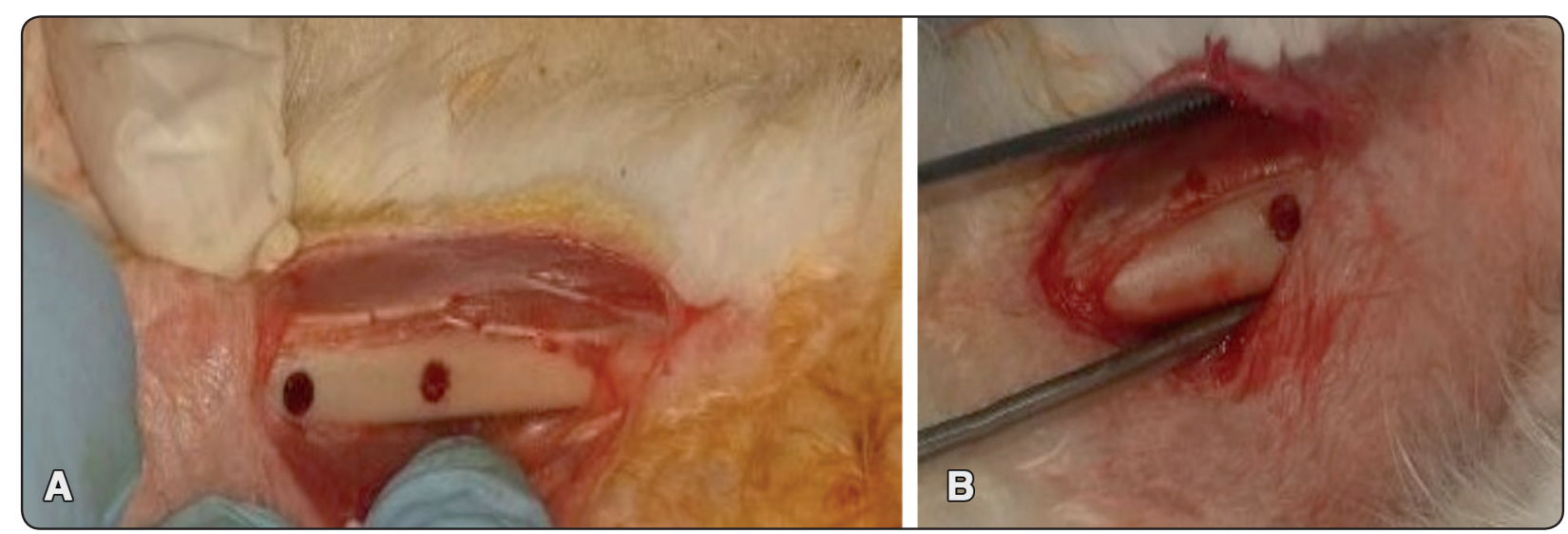

Fig. (2) a) Clinical view of surgically created defects in the right tibia. The first defect was grafted with leukocyte-platelet-rich fibrin (L-PRF). The other defect was left empty. b) Clinical view of surgically created defect in the left tibia. The defect was grafted with platelet-rich fibrin matrix (PRFM). 
$(18 \mathrm{~mm} \times 10 \mathrm{~mm})$ regions of interest $(\mathrm{ROI})$ on the scan images. Two ROI were positioned in the left tibia and one ROI was positioned in the right tibia. Results of BMD were expressed as grams per square centimeter.

\section{Histopathological examination:}

The animals were sacrificed at 10 and 21 days (4 animals at each time interval) via pentobarbital overdose $(100 \mathrm{mg} / \mathrm{kg})$. The tibias were carefully dissected free from soft tissues, and hard-tissue samples were fixed in $10 \%$ neutral buffered formalin for $48 \mathrm{~h}$ then decalcified in $10 \%$ ethylene diamine tetra-acetic acid (EDTA). Tissue processing, including dehydration, clearing, impregnation, and embedding, was done through graded ethanol, xylol, and paraffin. Histologic sections with a thickness of $6 \mu \mathrm{m}$ were prepared from each defect containing an intact border of the bone, and then the samples were routinely stained with hematoxylin and eosin (H\&E) staining ${ }^{(11)}$. For histological evaluation of healing process, Emery's histopathological healing criteria were used ${ }^{(12)}$, which are explained in Table 1. Histological examinations were performed by a single blinded investigator.

TABLE (1) Emery's histopathological healing criteria:

\begin{tabular}{|l|c|}
\hline \multicolumn{1}{|c|}{ Tissue present } & $\begin{array}{c}\text { Score } \\
\text { (point) }\end{array}$ \\
\hline Empty cavity & 0 \\
\hline Fibrous tissue only & 1 \\
\hline More fibrous tissue than fibrocartilage & 2 \\
\hline More fibrocartilage than fibrous tissue & 3 \\
\hline Fibrocartilage only & 4 \\
\hline More fibrocartilage than bone & 5 \\
\hline More bone than fibrocartilage & 6 \\
\hline Bone only & 7 \\
\hline
\end{tabular}

\section{RESULTS}

\section{Clinical evaluation}

All the surgical sites healed well. No signs of inflammation or infection were observed at any time point in any of the samples.

\section{BMD Analysis}

The PRFM group showed the highest BMD score at $10^{\text {th }}$ and 21 st days and the BMD score in the control group was the least (Fig. 3).

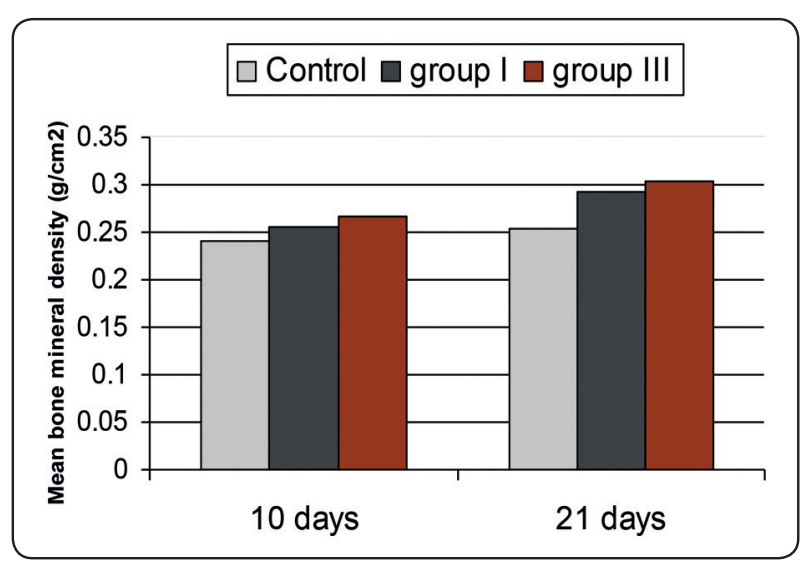

Fig. (3) DXA analysis of tibia bone.

\section{Histopathological findings}

Regarding to bone specimens collected after 10 days of surgery; control group (group I) revealed empty space (score 0) fig. 1-a. Group II treated by PRF showed activation of mesenchymal cells in the soft tissues. Bone surrounding the fracture gap differentiates into chondroblasts that make fibrocartilage and hyaline cartilage. More fibrous tissue than fibrocartilage (score 2) were seen fig. 1-b. On the other side group III treated by PRFM showed more fibrocartilage than fibrous tissue which characterized by well developed cartilaginous tissue with prominent basophilic matrix (score 3) fig. 1-c.

Histopathological findings of bone gap after 21 days of surgery; control group I showed more fibrocartilage than fibrous tissue (score 3) fig.2-a. 
In group II treated by PRF revealed small foci of chondrocytes proliferated in the bony tissues. In this group, the gap was filled with trabecular bone characterized by acellular osteocyte lacunae and fibrocartilage tissues (score 5) fig.2-b. Group III treated by PRFM showed that the newly formed cartilaginous tissue in the fracture gap undergoes enchondral ossification (score 7). The healed bone gap inhabited osteocyte lacunae and delimited by osteoblasts that forming a network of bone connected to the reactive trabeculae deposited elsewhere in the medullary cavity and beneath the periosteum fig. 2-c.

\section{DISCUSSION}

Fibrin-based membranes could be used as scaffolds for proliferation of periosteal and osseous cells. However, their clinical beneficial effects on bone regeneration need to be thoroughly explored. Although fibrin-based membranes have longer resorption time than the other platelet concentrates ${ }^{(13)}$, it has features to similar to those of natural clotting. Therefore, experimental evaluations investigating the early stages of healing would be beneficial.

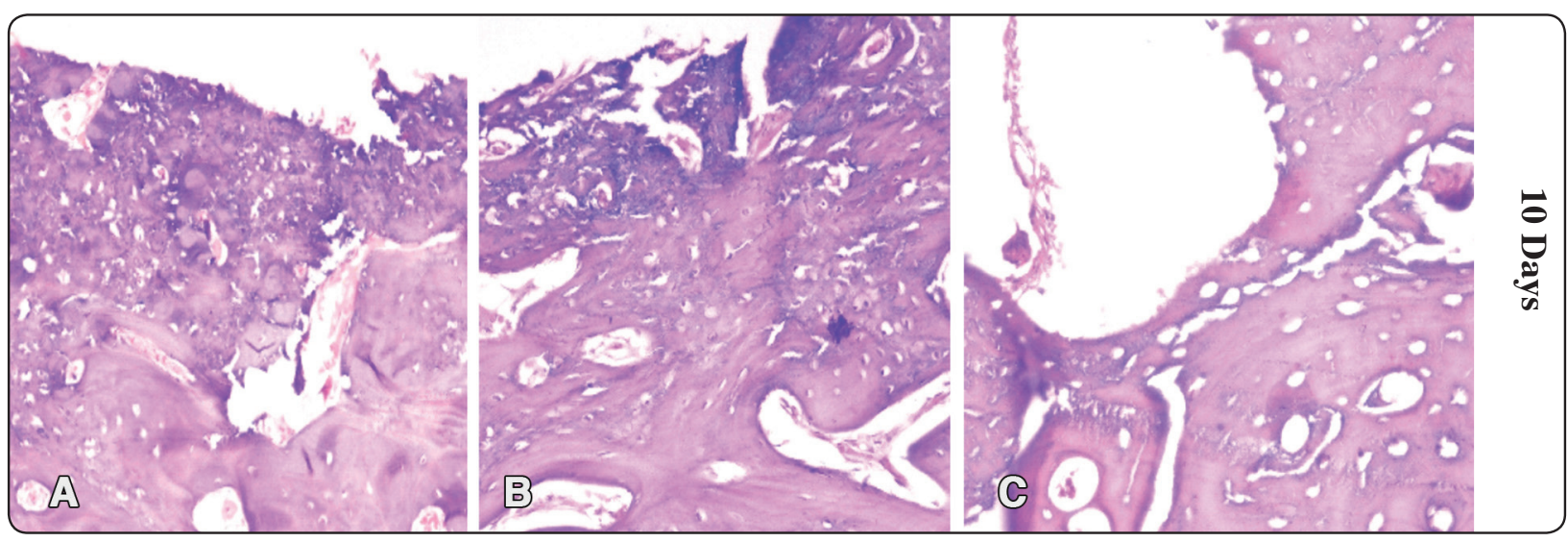

Fig. (4) Histopathological section of induced bone gap: control group showing empty gap (a), group treated by L-PRF showing more fibrous tissue than fibrocartilage (b), group treated by PRFM showing more fibrocartilage than fibrous tissue (c) (H\&E X200)

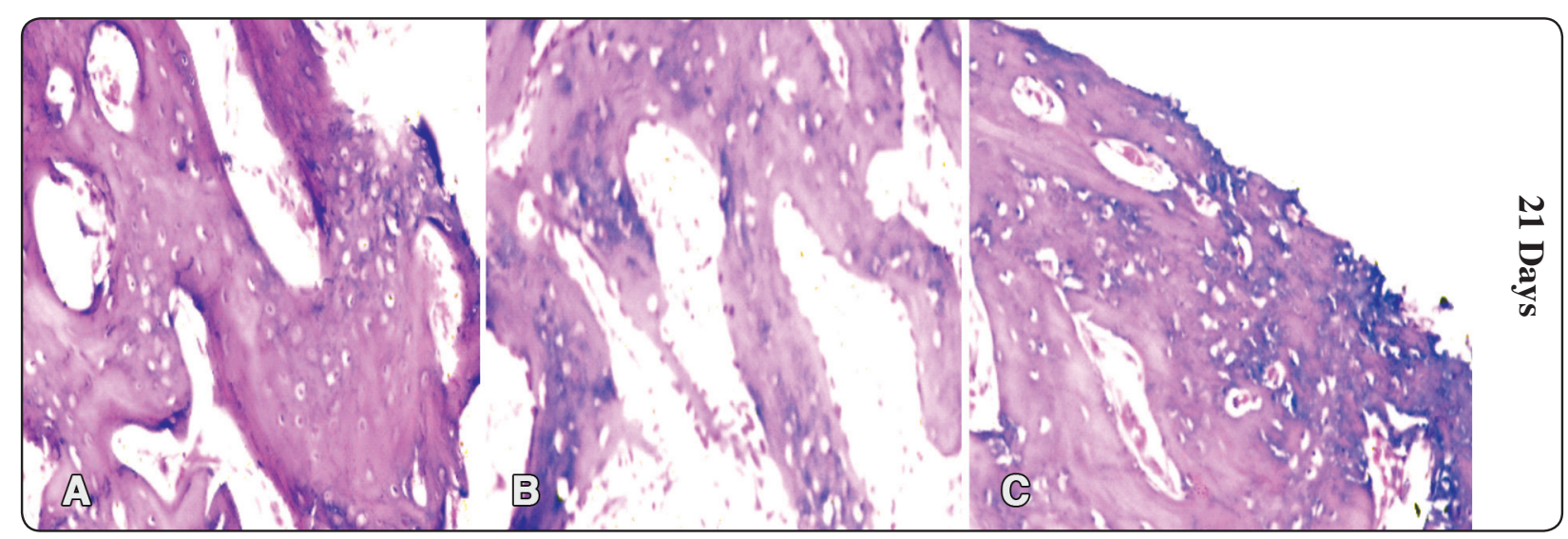

Fig. (5) Histopathological section of induced bone gap: control group showing more fibrocartilage than fibrous tissue arrow (a), group treated by L-PRF showing small foci of chondrocytes proliferated in the bony tissues arrow (b), group treated by PRFM showing enchondral ossification arrow (c) (H\&E X200). 
Histological samples at early steps in the bone regeneration process are needed to demonstrate the hypothetical beneficial effect of Fibrin-based membranes on bone regeneration. For these reasons, it is relevant to use a standardized animal. In the present study, we evaluated L-PRF in comparison to PRFM in surgically created experimental bone defects. The results showed more new bone formation in defects filled with PRFM than L-PRF and empty defects. Moreover, the BMD analysis showed higher bone density in PRFM and L-PRF groups than in the control group at 10 and 21 days evaluation period. The better results obtained with PRFM may be attributed to structural differences between PRFM and L-PRF which may be partially attributed to their differences in polymerization as a result of inducing extensive fibrin network formation through increased gravitational force during centrifugation.

The present study shows beneficial effects with adding fibrin based -membranes to surgically created defects in animal model. Better results was obtained with PRFM, however, the study had some limitations. The sample size was not large enough (4 animals in each group) to show statistically significant differences between groups and longer evaluation periods is needed to evaluate the effects on all stages of bone regeneration.

\section{REFERENCES}

1. Oda T., Kinshita and Ueda M.: effect of cortical bone perforation on periosteal Destruction. J Maxillofacial Surg 2009; 67 (7)1478-85.

2. ung RE, Glauser R, Scharer P, Hammerle CH, Sailer HF, Weber FE.Effect of rh-BMP-2 on guided bone regeneration in humans. Clin Oral Implants Research 2003; 14:556-68.

3. Nevins, M, Giannobile WV, McGuire MK, Kao RT, Mellonig JT, Hinrichs JT, et al. Plateletderived growth factor stimulates bone fill and rate of attachment level gain: Results of a large multicenter randomized controlled trial. J Periodontol 2005; 76:2205-15.
4. Bashutski JD, Wang HL. Periodontal and endodontic regeneration. J Endod 2009;35:321-328.

5. Dohan DM, Choukroun J, Diss A, Dohan SL, Dohan AJ, Mouhyi J, Gogly B. Platelet-rich fibrin (PRF): a secondgeneration platelet concentrate. Part I: technological concepts and evolution. Oral Surg Oral Med Oral Pathol Oral Radiol Endod 2006; 101:e37-

6. Kawase, T., Okuda, K., Saito, Y., and Yoshie, H. In vitro evidence that the biological effects of platelet-rich plasma on periodontal ligament cells is not mediated solely by constituent transforming-growth factor-beta or plateletderived growth factor. J Periodontol 76, 760, 2005.

7. Tajima, N., Sotome, S., Marukawa, E., and Omura, K. A three-dimensional cell-loading system using autologous plasma loaded into a porous b-tricalcium-phosphate block promotes bone formation at extraskeletal sites in rats. Mater Sci Eng 27, 625, 2007.

8. Dohan Ehrenfest DM, Bielecki T, Jimbo R, et al. Dothe fibrin architecture and leukocyte content influence the growth factor release of platelet concentrates? An evidence-based answer comparing a pure platelet-rich plasma (P-PRP) gel and a leukocyte-and platelet-rich fibrin (L-PRF) Curr Pharm Biotechnol. 2012;13:1145-1152.

9. Lucarelli E, Beretta R, Dozza B, Tazzari PL, O’Connell SM, Ricci F, Pierini M Squarzoni S, Pagliaro PP, Oprita EI, and Donati D. A recently developed bifacial plateletrich fibrin matrix. Eur J Cells Materials. 2010; 20:13-23.

10. Carroll RJ, Amoczky SP, Graham S, O'Connell SM. Characterization of autologous growth factors in Cascade platelet rich fibrin matrix (PRFM). Edison, NJ: Musculoskelatal Transplant Foundation 2005.

11. Bancroft, J.D., A. Stevens and D.R. Turner, (1996). Theory and Practice of Histological Techniques. $4^{\text {th }}$ Edn., Churchill, Livingston, New York, London, San Francisco, Tokyo.

12. Emery, S.E., Brazinski, M.S., Koka, A., Bensusan, J.S. and Stevenson, S. (1994). The Biological and Biomechanical Effects of Irradiation on Anterior Spinal Bone Grafts in a Canine Model, J. Bone Joint Surg. Am., 76-A (4): 540-548.

13. Clark RA.Fibrin and wound healing. Ann NYAcadSci. 2001; 936:355-367. 